\title{
Search for axioelectric effect of 5.5 MeV solar axions using BGO detectors
}

\author{
A.V. Derbin ${ }^{a}$, S.V. Bakhlanov, I.S. Dratchnev, A.S. Kayunov, V.N. Muratova \\ St. Petersburg Nuclear Physics Institute, Gatchina 188300, Russia
}

Received: 19 December 2012 / Revised: 10 April 2013 / Published online: 2 July 2013

(C) The Author(s) 2013. This article is published with open access at Springerlink.com

\begin{abstract}
A search for axioelectric absorption of solar axions produced in the $p+d \rightarrow{ }^{3} \mathrm{He}+\gamma(5.5 \mathrm{MeV})$ reactions has been performed with a BGO detector placed in a lowbackground setup. A model-independent limit on an axionnucleon and axion-electron coupling constant has been obtained: $\left|g_{A e} \times g_{A N}^{3}\right|<2.9 \times 10^{-9}$ for $90 \%$ confidence level. The constrains of the axion-electron coupling have been obtained for hadronic axion with masses in (0.1-1) MeV range: $\left|g_{A e}\right| \leq(1.4-9.7) \times 10^{-7}$.
\end{abstract}

\section{Introduction}

The axion is a pseudo-Goldstone boson arising from breaking the global Peccei-Quinn (PQ) symmetry [1-3]. The original so-called Weinberg-Wilczek-Peccei-Quinn axion model contained some certain direct predictions for the coupling constants between axions and photons $\left(g_{A \gamma}\right)$, electrons $\left(g_{A e}\right)$ and nucleons $\left(g_{A N}\right)$ as well as for the axion mass $\left(m_{A}\right)$. This model was quickly disproved by the reactor and accelerator experiments [4].

The particle has been retained by two new theoretical models of the "invisible" axion in the form required to solve the CP problem of strong interactions; at the same time its interaction with matter is suppressed. These are models of the "hadronic" (or Kim-Shifman-VainsteinZakharov (KSVZ)) [5, 6] and the "GUT" (or Dine-FischlerSrednicki-Zhitnitskii (DFSZ)) [7, 8] axion. The scale of Peccei-Quinn symmetry violation is arbitrary in both models and can be extended up to the Planck mass $\approx 10^{19} \mathrm{GeV}$. The axion mass in these models is determined by the axion decay constant $f_{A}$ :

$m_{A} \approx \frac{f_{\pi} m_{\pi} \sqrt{z}}{f_{A}(1+z)} ; \quad m_{A}(\mathrm{eV}) \approx \frac{6.0 \times 10^{6}}{f_{A}(\mathrm{GeV})}$,

a e-mail: derbin@pnpi.spb.ru where $m_{\pi}$ and $f_{\pi}$ are, respectively, the mass and decay constant of neutral $\pi$-meson and $z=m_{u} / m_{d}$ is quark-mass ratio. Since the axion coupling constants $g_{A \gamma}, g_{A e}$ and $g_{A N}$ are proportional to the axion mass, the interaction of an axion with matter is suppressed.

The main experimental efforts are focused on searches for an axion with a mass in the range of $10^{-6}$ to $10^{-2} \mathrm{eV}$. As this range is free of astrophysical and cosmological constraints, relic axions with such a mass could be considered to be the most likely candidates for dark matter particles. Experimental bounds on the mass of the axion follow from the constraints on the $g_{A \gamma}, g_{A e}$, and $g_{A N}$ coupling constants, which significantly depend on the theoretical model used.

New possibilities for solving a strong CP problem are based on the concept of the existence of the mirror particles [9] and supersymmetry [10]. These models suppose the existence of axions with the mass of about $1 \mathrm{MeV}$, and this existence is forbidden by neither laboratory experiments nor astrophysical data.

This work is devoted to the search for solar axions with an energy of $5.5 \mathrm{MeV}$, produced in the $p+d \rightarrow{ }^{3} \mathrm{He}+\mathrm{A}$ reaction. The range of axion masses under study is expanded up to $5 \mathrm{MeV}$. The axion flux is proportional to the $p p$-neutrino flux, which is estimated with a high degree of accuracy [11]. The axion interaction exploited in this study is the axioelectric effect $\mathrm{A}+e+Z \rightarrow e+Z$. As the cross section of the reaction depends on the charge of a nucleus as $Z^{5}$, the BGO detector containing bismuth (stable nucleus with the largest charge $Z=83$ ) is the most suitable from this point of view.

Recently, the high energy solar axions and axions from a nuclear reactor have been sought by the Borexino [12, 13], the CAST [14] and the Texono [15] collaborations. We have previously published a search for $5.5 \mathrm{MeV}$ axions with two small BGO detectors [16]. 


\section{Axion production in nuclear magnetic transitions and the axioelectric effect}

Since the temperature in the center of the Sun is about $1.3 \mathrm{keV}$, the Sun should be an intense source of low energy $\sim(1-10) \mathrm{keV}$ axions. The reactions of the main solar chain and $\mathrm{CNO}$ cycle can produce axions with higher energies. The most intensive flux is expected as a result of the reaction: $p+d \rightarrow{ }^{3} \mathrm{He}+\gamma$ when $5.5 \mathrm{MeV}$ axion is emitted instead $\gamma$-quantum.

According to the standard solar model (SSM), $99.7 \%$ of all deuterium is produced as a result of the two protons fusion, $p+p \rightarrow d+e^{+}+v_{e}$, while the remaining $0.3 \%$ is due to the $p+p+e^{-} \rightarrow d+v_{e}$ reaction. The expected solar axion flux can thus be expressed in terms of the $p p$-neutrino flux, which is $6.0 \times 10^{10} \mathrm{~cm}^{-2} \mathrm{~s}^{-1}$ [11]. The proportionality factor between the axion and neutrino fluxes is determined by the axion-nucleon coupling constant $g_{A N}$, which consists of isoscalar $g_{A N}^{0}$ and isovector $g_{A N}^{3}$ components.

In the $p\left(d,{ }^{3} \mathrm{He}\right) \gamma$ reaction, the M1-type transition corresponds to the capture of a proton with a zero orbital momentum. The probability of proton capture from the $S$ state at proton energies below $80 \mathrm{keV}$ has been measured in [17]; at the proton energy of $\sim 1 \mathrm{keV}, \mathrm{M} 1$ fraction of the total $p\left(d,{ }^{3} \mathrm{He}\right) \gamma$ cross section is $\chi=0.55$. The proton capture from the $S$ state corresponds to the isovector transition, and the ratio of the probability of a nuclear transition with the axion production $\left(\omega_{A}\right)$ to the probability of a magnetic transition $\left(\omega_{\gamma}\right)$ depends only on $g_{A N}^{3}[18-22]$ :

$\frac{\omega_{A}}{\omega_{\gamma}}=\frac{\chi}{2 \pi \alpha}\left[\frac{g_{A N}^{3}}{\mu_{3}}\right]^{2}\left(\frac{p_{A}}{p_{\gamma}}\right)^{3}=0.54\left(g_{A N}^{3}\right)^{2}\left(\frac{p_{A}}{p_{\gamma}}\right)^{3}$,

where $p_{\gamma}$ and $p_{A}$ are, respectively, the photon and axion momenta; $\alpha \approx 1 / 137$ is the fine-structure constant; and $\mu_{3}=\mu_{p}-\mu_{n} \approx 4.71$ is isovector nuclear magnetic momenta.

Within the hadronic axion model, the constant $g_{A N}^{3}$ can be written in terms of the axion mass [23, 24]:

$g_{A N}^{3}=-2.75 \times 10^{-8}\left(m_{A} / 1 \mathrm{eV}\right)$.

The value of $g_{A N}^{3}$ in the DFSZ model depends on an additional unknown parameter $\cos ^{2} \beta$, but have the same order of magnitude. The numerical value lies in the range of $(0.3-1.5)$ from the value of $g_{A N}^{3}$ for the hadronic axion [23].

The calculated values of the $\omega_{A} / \omega_{\gamma}$ ratio as a function of the axion mass are shown in Fig. 1. The axion flux on the Earth's surface is

$\Phi_{A}=\Phi_{v p p}\left(\omega_{A} / \omega_{\gamma}\right)$

where $\Phi_{v p p}=6.0 \times 10^{10} \mathrm{~cm}^{-2} \mathrm{~s}^{-1}$ is the $p p$-neutrino flux.

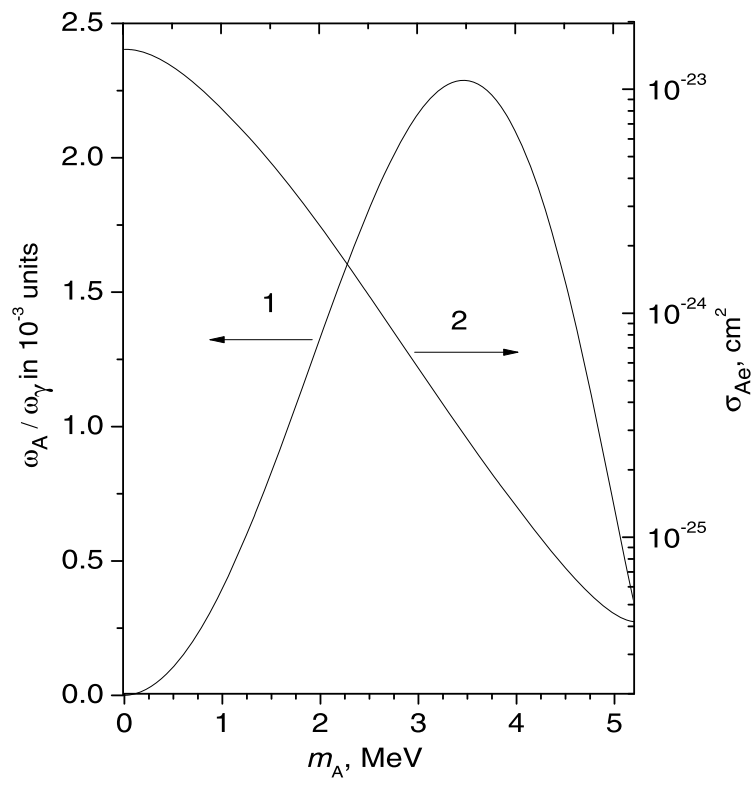

Fig. 1 The ratio of the emission probabilities for axions and $\gamma$ quanta $\left(\omega_{A} / \omega_{\gamma}\right)$ in the $p+d \rightarrow{ }^{3} \mathrm{He}+\gamma$ reaction (curve 1, left-hand scale); the cross section of the axioelectric effect for $5.5-\mathrm{MeV}$ axions on bismuth atoms for $g_{A e}=1$ (curve 2, right-hand scale)

To detect 5.5 MeV axions, we chose the reaction of axioelectric effect $A+Z+e \rightarrow Z+e$ which is caused by the axion-electron interaction. The cross section of the axioelectric effect depends on the nuclear charge according to the $Z^{5}$ law, and therefore, it is reasonable to search for this process using detectors with a large $Z$. The cross section for bismuth atoms exceeds the cross section of the Compton conversion of an axion $\left(A+e^{-} \rightarrow \gamma+e^{-}\right)$by almost two orders of magnitude. The detection efficiency for the produced electron is almost $100 \%$ and the background level at $5.5 \mathrm{MeV}$ is much lower than in the range of natural radioactivity. As a result, the sensitivity to constants $g_{A e}$ and $g_{A N}$ can be high even in an experiment using a relatively small target mass.

In the axioelectric effect (an analog of the photoelectric effect), an axion disappears and an electron with the energy of $E_{e}=E_{A}-E_{b}$, where $E_{b}$ is the electron binding energy, is emitted from the atom. The axioelectric effect cross section for K-shell electrons has been calculated (on the assumption that $E_{A} \gg E_{b}$ and $\left.Z \ll 137\right)$ in [25]:

$$
\begin{aligned}
\sigma_{A e}= & 2\left(Z \alpha m_{e}\right)^{5} \frac{g_{A e}^{2}}{m_{e}^{2}} \frac{p_{e}}{p_{A}}\left[\frac{4 E_{A}\left(E_{A}^{2}+m_{A}^{2}\right)}{\left(p_{A}^{2}-p_{e}^{2}\right)^{4}}-\frac{2 E_{A}}{\left(p_{A}^{2}-p_{e}^{2}\right)^{3}}\right. \\
& -\frac{64}{3} p_{e}^{2} p_{A}^{2} m_{e} \frac{m_{A}^{2}}{\left(p_{A}^{2}-p_{e}^{2}\right)^{6}}-\frac{16 m_{A}^{2} p_{A}^{2} E_{e}}{\left(p_{A}^{2}-p_{e}^{2}\right)^{5}} \\
& \left.-\frac{E_{A}}{p_{e} p_{A}} \frac{1}{\left(p_{A}^{2}-p_{e}^{2}\right)^{2}} \ln \frac{p_{e}+p_{A}}{p_{e}-p_{A}}\right] .
\end{aligned}
$$


The dependence of the cross section on the axion mass for the coupling constant $g_{A e}=1$ is shown in Fig. 1. The $\mathrm{K}$-shell electrons make the main contribution to the cross section. The contribution from the other electrons was incorporated by introducing a factor of $5 / 4$, by analogy with the photoelectric effect.

\section{Interaction of axions with solar matter and axion decays}

The flux of $5.5 \mathrm{MeV}$ axions on the Earth's surface is proportional to the $p p$-neutrino flux only when the axion lifetime exceeds the time of a flight from the Sun and when the flux is not reduced as a result of the axion absorption by matter inside the Sun. The requirement that most axions escape the Sun and reach the Earth thus limits the axion coupling strengths accessible to terrestrial experiments $[12,13,16,19]$.

Axions leaving the center of the Sun pass through the matter layer of $\approx 6.8 \times 10^{35}$ electrons and $\approx 5 \times 10^{35}$ protons per $\mathrm{cm}^{-2}$ in order to reach the Sun's surface. The maximum cross section of the axio-electric effect on atoms for $5.5 \mathrm{MeV}$ axions is $\sigma_{A e} \approx g_{A e}^{2} Z^{5} 3.8 \times 10^{-33} \mathrm{~cm}^{2}$ (see Fig. 1 for $\mathrm{Bi}$ ). Axion loss due to axioelectric absorption by $\mathrm{Fe}$ atoms $\left(Z=26, N_{\mathrm{Fe}} / N_{\mathrm{H}}=2.8 \times 10^{-5}\right)$ imposes an upper limit on $\left|g_{A e}\right|<10^{-4}$ above which the sensitivity of terrestrial experiments using solar axions is reduced. The abundance of heavy $(Z>50)$ elements in the Sun is $N_{Z} / N_{\mathrm{H}} \sim$ $10^{-9}$ in relation to hydrogen [26]. If $\left|g_{A e}\right|<10^{-3}$, the change in the axion flux does not exceed $10 \%$.

The other process-Compton conversion of an axion into a photon-imposes stronger limit on the sensitivity of Earthbound experiments to the constant $g_{A e}$. The cross section of this reaction for $5.5-\mathrm{MeV}$ axions depends weakly on the axion mass and can be written as $\sigma_{c c} \approx g_{A e}^{2} 4 \times 10^{-25} \mathrm{~cm}^{2}$ $[13,18]$. For $\left|g_{A e}\right|$ values below $10^{-6}$, the axion flux is not substantially suppressed.

The axion-photon interaction, as determined by the constant $g_{A \gamma}$, leads to the conversion of an axion into a photon in a field of nucleus (Primakoff conversion). The cross section of the reaction is $\sigma_{p c}(5.5 \mathrm{MeV}) \approx g_{A \gamma}^{2} Z^{2} 2 \times$ $10^{-29} \mathrm{~cm}^{2}$. Taking into account the density of protons and ${ }^{4} \mathrm{He}$ nuclei, the condition that axions efficiently escape the Sun imposes the constraint $\left|g_{A \gamma}\right|<10^{-4} \mathrm{GeV}^{-1}$. Constraint for the other elements are negligible due to their low concentration in the Sun.

The axion-nucleon interaction leads to axion absorption in a threshold reaction similar to photo-dissociation: $A+$ $Z \rightarrow Z_{1}+Z_{2}$. For axions with energy $5.5-\mathrm{MeV}$ this can occur for only a few nuclei: ${ }^{17} \mathrm{O},{ }^{13} \mathrm{C},{ }^{3} \mathrm{He}$ and ${ }^{2} \mathrm{H}$ [19]. It was shown in [19] that the absorption of axions can be mainly due to the process $A+{ }^{17} \mathrm{O} \rightarrow{ }^{16} \mathrm{O}+n$. The cross section of the reaction depends on the combination of iso-scalar and iso-vector coupling constants. If $\left|-g_{A N}^{0}+g_{A N}^{3}\right|<10^{-2}$, the change in the axion flux does not exceed $10 \%$. Because the absorption of axions due to isovector-transition $A\left({ }^{3} \mathrm{He}, d\right) p$ is insignificant, the range of values $\left|g_{A N}^{3}\right|$ available for the study is practically unlimited.

For the axions with a mass above $2 m_{e}$, the main decay mode is the decay into an electron-positron pair. The condition that $90 \%$ of all axions reach the Earth limits the sensitivity of the solar axion experiments to $\left|g_{A e}\right|<$ $\left(10^{-12}-10^{-11}\right)$ [16]. On the other hand the measured value of the interplanetary positron flux allowed us to establish a new constraint on the axion-electron coupling constant: $g_{A e} \leq(1-5) \times 10^{-17}$ for axions with masses in the range of (1.2-5.4) MeV [16].

If the axion mass is less than $2 m_{e}, A \rightarrow e^{+}+e^{-}$decay is forbidden, but the axion can decay into two $\gamma$ quanta. The probability of the decay depends on the axionphoton coupling constant and the axion mass- $\tau_{A \rightarrow \gamma \gamma}=$ $64 \pi / g_{A \gamma}^{2} m_{A}^{3}$. The present-day experimental constraint on $g_{A \gamma}$ is $10^{-9} \mathrm{GeV}^{-1}$, which corresponds to $\tau_{c m}=10^{5} \mathrm{~s}$ for $1-\mathrm{MeV}$ axions. This means that the axion flux is not practically reduced due to the $A \rightarrow 2 \gamma$ decay even for axions with $5 \mathrm{MeV}$ mass.

\section{Experimental setup}

We used a $2.46 \mathrm{~kg}$ BGO crystal, manufactured from bismuth orthogermanate $\mathrm{Bi}_{4} \mathrm{Ge}_{3} \mathrm{O}_{12}(1.65 \mathrm{~kg}$ of $\mathrm{Bi})$, to search for the $5.5 \mathrm{MeV}$ axions. The BGO crystal was grown at the Nikolaev Institute of Inorganic Chemistry and it was shaped as a cylinder, $76 \mathrm{~mm}$ in diameter and $76 \mathrm{~mm}$ in height. The detector signal was measured by an R2887 photoelectron multiplier, which had an optical contact with a crystal end surface.

The spectrometric channel of the BGO scintillation detector included an amplifier with the shaping time of $1 \mu \mathrm{s}$ and a 12-digit ADC. The amplification was selected so that the ADC channel scale was $2.7 \mathrm{keV}$. The standard calibration sources $\left({ }^{60} \mathrm{Co}\right.$ and $\left.{ }^{207} \mathrm{Bi}\right)$, in combination with the natural radioactivity lines of ${ }^{40} \mathrm{~K}$ and the uranium and thorium families, were used for the energy calibration of the detector. The energy dependence of the detector resolution $\sigma$ can be presented as $\sigma / E \approx 3.9 \% \times E^{-1 / 2}$, where $E$ is in $\mathrm{MeV}$. The detection efficiency $\varepsilon$ for $5.5-\mathrm{MeV}$ electrons in the BGO crystal was calculated with GEANT4. The response function consists of Gaussian peak and a flat tail, the number of events in Gaussian peak is $\varepsilon=0.67$.

The external $\gamma$ activity was suppressed using a passive shield that consisted of successive layers of lead $(90 \mathrm{~mm})$ and bismuth $\left(15 \mathrm{~mm} \mathrm{Bi}_{2} \mathrm{O}_{3}\right)$. The total thickness of the passive shield was $\approx 110 \mathrm{~g} \mathrm{~cm}^{-2}$. 


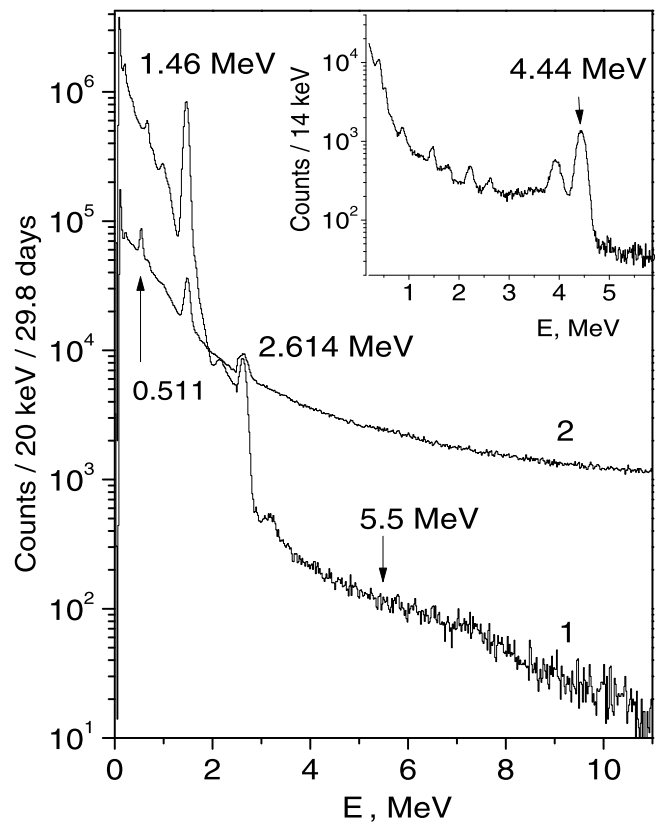

Fig. 2 The energy spectrum of the BGO detector measured (1) in anticoincidence and (2) in coincidence with the active shielding signal. The location of the expected $5.5 \mathrm{MeV}$ axion peak is denoted by an arrow. In inset the spectrum measured with $\mathrm{Pu}-\mathrm{Be}$ neutron source is shown

The setup was located on the Earth's surface. In order to suppress the cosmic-ray background we used an active veto, which consisted of five $50 \times 50 \times 12 \mathrm{~cm}$ plastic scintillators. The active veto included two energy thresholds: the first was set at a level $\sim 5 \mathrm{MeV}$, which corresponds to $600 \mathrm{~s}^{-1}$ counts rate and $4 \%$ dead time for $70 \mu$ s inhibit pulse. The second stage with $\sim 0.1 \mathrm{MeV}$ threshold and $\pm 6 \mu$ s inhibit pulse preformed a selection among the BGO impulses for energies exceeding 2.3 MeV. This energy limit was established in order to make an estimate of the second stage dead time using the $2.614 \mathrm{MeV}$ natural radioactivity peak. Our estimate to the active shielding dead time is $23 \%$.

\section{Results}

The measurements were performed over 29.8 days in live time by 2 -hour series. The measurements were divided into series in order to monitor the time stability of the BGO detector and the active shielding. The energy spectrum of the BGO detector in the range of (0-11) MeV is shown in Fig. 2. The spectrum of the BGO signals that were not accompanied by the active shielding signal is designated as 1 .

In the spectrum, one can identify two pronounced peaks at $1.460 \mathrm{MeV}$ and $2.614 \mathrm{MeV}$; these are due to the natural radioactivity of the ${ }^{40} \mathrm{~K}$ (located in the PMT's glass housing) and of ${ }^{208} \mathrm{Tl}$ from the ${ }^{232} \mathrm{Th}$ family (Fig. 2). The positions and intensities of these peaks were used for monitoring of time stability.
Bismuth has the largest nuclear charge among the stable isotopes $(Z=83)$, and the cross section of $\left(e^{+} e^{-}\right)$-pair production upon the interaction of $\gamma$ quanta is therefore the largest for this element. The annihilation peak at $0.511 \mathrm{MeV}$ is pronounced in the spectrum. The peak at $2.10 \mathrm{MeV}$ is related to the escape of one annihilation $\gamma$ quantum from the detector upon the detection of $2.614 \mathrm{MeV} \gamma$ rays. The visible kink at $\approx 7.5 \mathrm{MeV}$ is due to the $\gamma$ quanta produced as a result of the capture of thermal neutrons by the components of the passive shield.

The positions and dispersion of the $1.46 \mathrm{MeV}$ and $2.614 \mathrm{MeV}$ peaks determined during the measurements were used to find the energy scale and resolution of the BGO detector. The energy calibration of the spectrometric channel was found as a linear function: $E=A \times N+B$, where $E$ is the released energy and $N$ is the channel number. For higher energies the energy calibration was checked with a ${ }^{239} \mathrm{Am}-{ }^{9} \mathrm{Be}$ neutron source. The reaction ${ }^{9} \mathrm{Be}(\alpha, n){ }^{12} \mathrm{C}^{*}$ produce $\gamma$-quanta with energy $4.439 \mathrm{MeV}$ corresponding to the energy of the first exited states ${ }^{12} \mathrm{C}$ nuclei. The position of $4.439 \mathrm{MeV}$ peak is restored with accuracy better than $5 \mathrm{keV}$ while $1461 \mathrm{keV}$ and $2614 \mathrm{keV}$ calibration peaks are used (Fig. 2, inset).

The dependence of the energy resolution of a scintillation detector vs energy can be written as $\sigma=C \times \sqrt{E}$. The parameter $\mathrm{C}$ was found to be $0.04 \mathrm{MeV}^{1 / 2}$. The values of $\sigma$ determined from the background spectrum are in good agreement with the measurements performed with ${ }^{60} \mathrm{Co},{ }^{207} \mathrm{Bi}$ and ${ }^{239} \mathrm{Pu}^{9} \mathrm{Be}$ standard calibration sources. The expected standard deviation of the $5.5 \mathrm{MeV}$ peak due to the axion absorption is $\sigma=93 \mathrm{keV}$.

Figure 3 shows the energy range of (4.5-6.5) $\mathrm{MeV}$, in which the axion peak was expected. The spectrum measured in the range of (4.5-6.5) MeV was fitted by a sum of exponential and two Gaussian functions:

$N(E)=a+b \times \exp (c E)+\sum_{i=1}^{2} \frac{S_{i}}{\sqrt{2 \pi \sigma_{i}}} \exp \left[-\frac{\left(E_{i}-E\right)^{2}}{2 \sigma_{i}^{2}}\right]$.

Here $a, b$ and $c$ are parameters of the function describing the smooth background. The position and dispersion of the first Gaussian peak corresponded to the desired-peak parameters: $E_{1}=5.49 \mathrm{MeV}$ is the axion peak position, $\sigma_{1}=0.093 \mathrm{MeV}$ is the Gaussian peak standard deviation.

Because a small unknown peak can be seen at $\approx 5.8 \mathrm{MeV}$, the second Gaussian was added to the fitting function. The position and area of the second peak were free, while the dispersion $\sigma_{2}=0.095 \mathrm{MeV}$ was fixed.

The position of first peak $\left(E_{1}\right)$ and dispersion $\left(\sigma_{1}\right)$ were fixed and six parameters were varied, three of which described the continuous background while three others described area of the two peaks $\left(S_{1}, S_{2}\right)$, and the second peak 


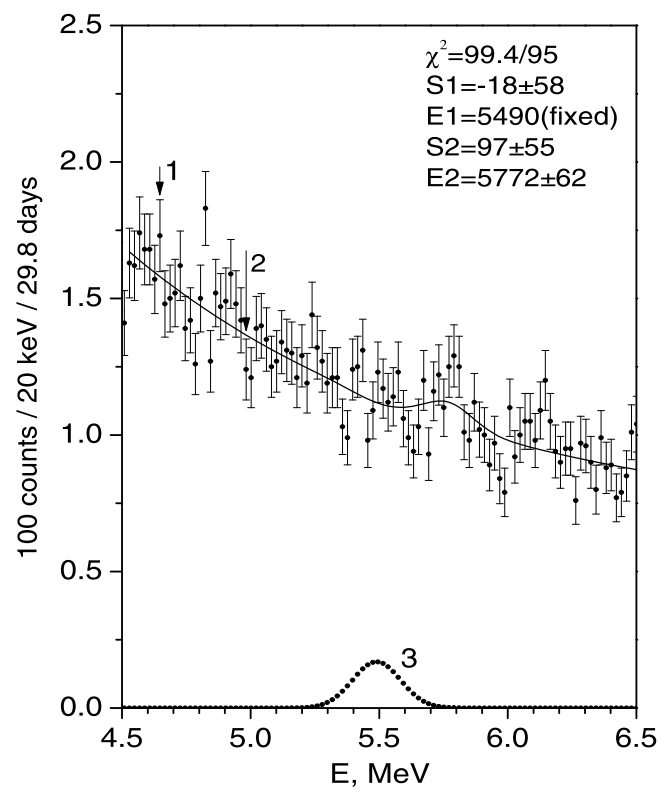

Fig. 3 The fitted BGO spectrum in the (4.5-6.5) $\mathrm{MeV}$ range. Curve 3 is the detector response function for $E_{0}=5.49 \mathrm{MeV}$ and $\sigma=0.093 \mathrm{MeV}$

position $\left(E_{2}\right)$. The total number of the degrees of freedom in the range of (4.5-6.5) MeV was 95.

The fit results, corresponding to the minimum $\chi^{2}=$ 99.4/95, are shown in Fig. 3. The position and area of the second peak are: $E_{2}=5.77 \pm 0.06 \mathrm{MeV}$ and $S_{2}=97 \pm 55$ counts. We attribute this peak to the intense $5.824 \mathrm{MeV}$ gamma-ray line resulting in the capture of thermal neutrons by ${ }^{113} \mathrm{Cd}$ [27]. The cross section of thermal neutron capture is $2 \times 10^{4}$ barns. The intensity of the $5.49 \mathrm{MeV}$ peak was found to be $S_{1}=-18 \pm 58$, this corresponds to the upper limit on the number of counts in the peak, $S_{\mathrm{lim}}=85$ at a $90 \%$ confidence level [28].

The expected number of axioelectric absorption events are:

$S_{a b s}=\varepsilon N_{\mathrm{Bi}} T \Phi_{A} \sigma_{A e}$

where $\sigma_{A e}$ is the axioelectric effect cross section, given by expression (5); $\Phi_{A}$ is the axion flux (4); $N_{\mathrm{Bi}}=4.76 \times 10^{24}$ is the number of $\mathrm{Bi}$ atoms; $T=2.57 \times 10^{6} \mathrm{~s}$ is the measurement time; and $\varepsilon=0.67$ is the detection efficiency for $5.5 \mathrm{MeV}$ electrons. The axion flux $\Phi_{A}$ is proportional to the constant $\left(g_{A N}^{3}\right)^{2}$, and the cross section $\sigma_{A e}$ is proportional to the constant $g_{A e}^{2}$, according to expressions (4) and (5). As a result, the $S_{a b s}$ value depends on the product of the axion-electron and axion-nucleon coupling constants: $\left(g_{A e}\right)^{2} \times\left(g_{A N}^{3}\right)^{2}$.

The experimentally found condition $S_{a b s} \leq S_{\text {lim }}$ imposes some constraints on the range of possible $\left|g_{A e} \times g_{A N}^{3}\right|$ and $m_{A}$ values. The range of excluded $\left|g_{A e} \times g_{A N}^{3}\right|$ values is

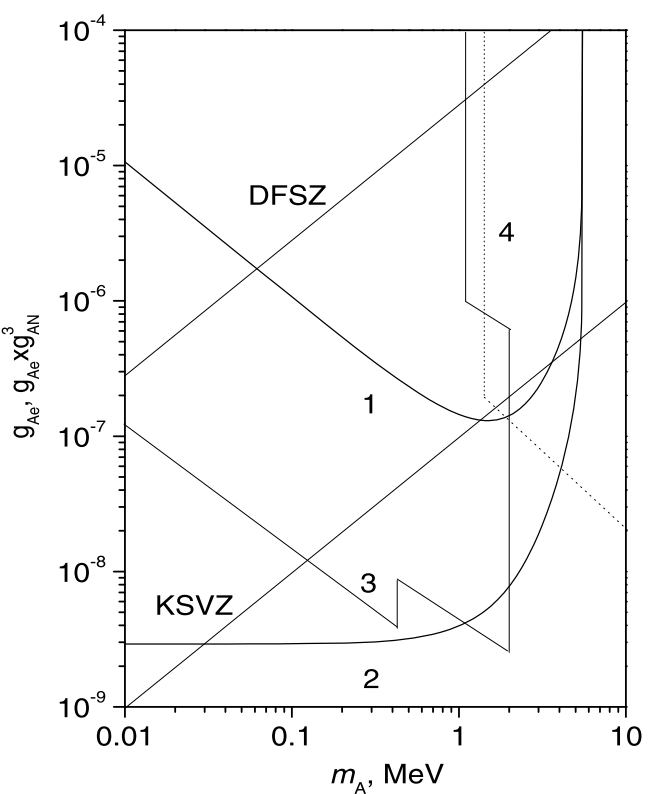

Fig. 4 The limits on the $g_{A e}$ coupling constant obtained by 1 -present work, 2-present work for $\left|g_{A e} \times g_{A N}^{3}\right|, 3$ - solar [12] and reactor experiments [15, 29], 4-beam dump experiments [30, 31]. The allowed $\left|g_{A e}\right|$ and $\left|g_{A e} \times g_{A N}^{3}\right|$ values lie below the corresponding curves. The relations between $g_{A e}$ and $m_{A}$ for DFSZ- and KSVZ-models are also shown

shown in Fig. 4, at $m_{A} \rightarrow 0$ the limit is

$\left|g_{A e} \times g_{A N}^{3}\right| \leq 2.9 \times 10^{-9}$.

The dependence of $\left|g_{A e} \times g_{A N}^{3}\right|$ on $m_{A}$ is related only to the kinematic factor in formulae (2) and (5). These constraints are completely model-independent and valid for any pseudoscalar particle with coupling $\left|g_{A e}\right|$ less than $10^{-6(4)}$.

Within the hadronic axion model, $g_{A N}^{3}$ and $m_{A}$ quantities are related by expression (3), which can be used to obtain a constraint on the $g_{A e}$ constant, depending on the axion mass (Fig. 4). For $m_{A}=1 \mathrm{MeV}$, this constraint corresponds to $\left|g_{\text {Ae }}\right| \leq 1.4 \times 10^{-7}$.

Figure 4 also shows the constraints on the constant $\left|g_{A e}\right|$ that were obtained in the Borexino experiment for $478-\mathrm{keV}$ ${ }^{7} \mathrm{Li}$ solar axions [12] and in the Texono reactor experiment for 2.2- $\mathrm{MeV}$ axions produced in the $n+p \rightarrow d+A$ reaction [15]. Recently, Borexino coll. reported new more stringent limits on $g_{A e}$ coupling for $5.5 \mathrm{MeV}$ solar axions [13]. Unlike our work, these limits on $g_{A e}$ were obtained in assumption that the axion interacts with electron through the Compton conversion process.

The sensitivity of the experiment to the constant $g_{A e}$ depends on the target mass $M$, specific background level $B$, detector resolution $\sigma$, and measurement time $T: g_{A e}^{\lim } \sim$ $(\sigma B / M T)^{1 / 2}$. The level $g_{A e} \approx 10^{-10}$ can be achieved with longer measurements with the detector mass enlarged by three orders of magnitude and the background level reduced 
by two orders of magnitude, the latter can be done by placing the setup in an underground laboratory.

\section{Conclusions}

A search for the axioelectric absorption of $5.5 \mathrm{MeV}$ axions produced in the $p+d \rightarrow{ }^{3} \mathrm{He}+\gamma$ reaction was performed using a BGO detector with a mass of $2.5 \mathrm{~kg}$, located in a low-background setup equipped with passive and active shielding. As a result, a model-independent limit on axionnucleon and axion-electron coupling constant has been obtained: $\left|g_{A e} \times g_{A N}^{3}\right|<2.9 \times 10^{-9}$ (90\% c.1.). Within the hadronic axion model the constraints on the axion-electron coupling constant $\left|g_{A e}\right| \leq(1.4-9.7) \times 10^{-7}$ for axions with masses $0.1<m_{A}<1 \mathrm{MeV}$ were obtained for $90 \%$ c.l.

Acknowledgements This work was supported by RFBR grants 1302-01199-a and 13-02-12140-ofi-m.

Open Access This article is distributed under the terms of the Creative Commons Attribution License which permits any use, distribution, and reproduction in any medium, provided the original author(s) and the source are credited.

\section{References}

1. S. Weinberg, Phys. Rev. Lett. 40, 223 (1978)

2. F. Wilczek, Phys. Rev. Lett. 40, 279 (1978)

3. R.D. Peccei, H.R. Quinn, Phys. Rev. Lett. 38, 1440 (1977)
4. J. Beringer et al. (Particle Data Group), Phys. Rev. D 86, 010001 (2012)

5. J.E. Kim, Phys. Rev. Lett. 43, 103 (1979)

6. M.A. Shifman, A.I. Vainstein, V.I. Zakharov, Nucl. Phys. B 166, $493(1980)$

7. A.R. Zhitnitskii, Yad. Fiz. 31, 497 (1980). Sov. J. Nucl. Phys., 31, 260, 1980

8. M. Dine, F. Fischler, M. Srednicki, Phys. Lett. B 104, 199 (1981)

9. Z. Berezhiani et al., Phys. Lett. B 500, 286 (2001)

10. L.J. Hall, T. Watari, Phys. Rev. D 70, 115001 (2004)

11. A.M. Serenelli, W.C. Haxton, C. Peña-Garay. arXiv:1104.1639

12. G. Bellini et al. (Borexino Collaboration), Eur. Phys. J. C 54, 61 (2008)

13. G. Bellini et al. (Borexino Collaboration), Phys. Rev. D 85, 092003 (2012)

14. S. Andriamonje et al. (CAST Collaboration), J. Cosmol. Astropart. Phys. 1003, 032 (2010). arXiv:0904.2103

15. H.M. Chang et al. (Texono Collaboration), Phys. Rev. D, 75, 052004 (2007)

16. A.V. Derbin et al., Bull. Russ. Acad. Sci., Phys. 74, 805 (2010). arXiv: 1007.3387

17. G.J. Schmid et al., Phys. Rev. C 56, 2565 (1997)

18. T.W. Donnelly et al., Phys. Rev. D 18, 1607 (1978)

19. G. Raffelt, L. Stodolsky, Phys. Lett. B 119, 323 (1982)

20. F.T. Avignone III. et al., Phys. Rev. D 37, 618 (1988)

21. W.C. Haxton, K.Y. Lee, Phys. Rev. Lett. 66, 2557 (1991)

22. A.V. Derbin et al., JETP Lett. 65, 576 (1997)

23. M. Srednicki, Nucl. Phys. B 260, 689 (1985)

24. D.B. Kaplan, Nucl. Phys. B 260, 215 (1985)

25. A.R. Zhitnitskii, Yu.I. Skovpen, Yad. Fiz. 29, 995 (1979)

26. M. Asplund, N. Grevesse, J. Sauval, Nucl. Phys. A 777, 1 (2006)

27. J. Blachot, Nucl. Data Sheets 104, 791 (2005)

28. G.J. Feldman, R. Cousins, Phys. Rev. D 57, 3873 (1998)

29. M. Altmann et al., Z. Phys. C 68, 221 (1995)

30. A. Konaka et al., Phys. Rev. Lett. 57, 659 (1986)

31. J.D. Bjorken et al., Phys. Rev. D 38, 3375 (1988) 